\title{
Lack of Agreement on Distal Radius Fracture Treatment
}

\author{
Katherine K. Bruce, BS, Daniel J. Merenstein, MD, Michael V. Narvaez, MD, \\ Steven K. Neufeld, MD, Megan J. Paulus, MD, Tina P. Tan, BA, \\ Jake R. Braunstein, MD, Ashley C. Pfaff, MD, Chun-Chib Huang, PhD, and \\ Robert B. Friedland, PhD
}

Introduction: Variation in clinical practice resulting from the absence of evidence-based treatment protocols has negative implications on both the cost and the quality of medical care. The objective of this study was to assess whether a standard of care for the treatment of extra-articular nondisplaced distal radius fracture has developed despite the lack of a conclusive recommendation from the American Academy of Orthopaedic Surgeons.

Methods: A case-vignette survey was conducted. Treatment type and duration of casting selections were analyzed. The cost implications of responses were assessed. Participants were practicing orthopedists primarily in the mid-Atlantic region of the United States. Orthopedists $(n=494)$ were recruited via E-mail and at the American Academy of Orthopaedic Surgeons Annual Meeting held in Chicago in March 2013. Inclusion criteria required that participants be graduates of an accredited medical school and be practicing orthopedists at the time of survey distribution. The main outcome measure was surgical or nonsurgical intervention.

Results: Nonsurgical treatment was selected by $60 \%$ of respondents, with surgery preferred by $37 \%$. Duration of casting responses varied from 2 to 12 weeks. Among nonsurgical responses, 69\% indicated 6 weeks as their preferred duration of casting (95\% confidence interval, 64.9-73.1\%). Surgery imposes a $76 \%$ greater total cost to society than nonsurgical treatments.

Conclusions: Our findings suggest the absence of a consensus strategy for the treatment of extraarticular nondisplaced distal radius fractures. Implications of variance in treatment on cost and quality support the need for established, evidence-based guidelines or further clinical trials to assist in the management of this common fracture. (J Am Board Fam Med 2016;29:218-225.)

Keywords: Bone Fractures, Cost-Effectiveness, Evidence-Based Medicine

Over 640,000 distal radius fracture cases were reported in the United States in 2001, making this one of the most common fractures seen in emergency

This article was externally peer reviewed.

Submitted 24 July 2015; revised 22 October 2015; accepted 28 October 2015.

From the Department of Human Science, Georgetown University School of Nursing and Health Studies, Washington, DC (KKB); the Department of Family Medicine, Georgetown University Medical Center, Washington, DC (DJM, TPT); Georgetown University School of Medicine, Washington, DC (MVN, JRB, ACP); the Orthopaedic Foot \& Ankle Center of Washington, Falls Church, VA (SKN); the Department of Orthopaedics, MedStar Georgetown University Hospital, Washington, DC (MJP); the Department of Biostatistics and Epidemiology, MedStar Health Research Institute, Hyattsville, MD (C-CH); and the Department of Health Systems Administration, Georgetown University School of Nursing and Health Studies, Washington, DC (RBF). departments. ${ }^{1}$ Distal radius fractures are prevalent across all age groups, with major peaks in incidence occurring among children aged 5 to 14 years, males $<50$ years old, and females $>40$ years old. ${ }^{2}$ In younger populations, radial fractures result primarily from local trauma caused by sports or motor vehicle accidents, whereas injuries among the elderly typically represent low-energy fractures. ${ }^{3}$

Funding: The authors received financial support for travel and space at the American Academy of Orthopaedic Surgeons conference in Chicago from Vilex and Merete.

Conflict of interest: none declared.

Corresponding author: Daniel J. Merenstein, MD, Georgetown University Medical Center, 4000 Reservoir Rd NW, Building D 240, Washington, DC 20007 (E-mail: djm23@georgetown.edu). 
Current treatment options for distal radius fractures range from conservative, nonoperative approaches to surgical reduction and fixation. These options include closed reduction with splint or cast immobilization, external fixation, percutaneous pinning, open reduction with internal fixation, and replacement of lost bone with a bone graft. ${ }^{3}$ There is currently no evidence-based treatment protocol for extra-articular nondisplaced distal radius fractures, as indicated by the American Academy of Orthopaedic Surgeons' (AAOS) inability to denote any of their 2009 recommendations as "strong" based on sufficient supporting evidence. ${ }^{3}$ In addition, the literature suggests a lack of consensus regarding optimal immobilization duration for sufficient healing and restoration of function following fractures of the distal radius. ${ }^{4-6}$

Without an evidence-supported consensus treatment protocol, physicians must rely on ideology, habit, or other methods to make treatment decisions that fuel clinical inconsistencies. ${ }^{7-9}$ This clinical variation in and absence of evidence for medical procedures have been associated with negative implications on both the cost and quality of medical care. ${ }^{8,9}$ We hypothesized that an informal standard of care would exist for distal radius fractures, and if variations did exist, they would primarily be the result of regional differences in accepted standards. Our objective was to assess the current practice environment to identify similar practice patterns resulting from an informal standard of care.

\section{Methods}

\section{Study Design}

Orthopedic surgeons were surveyed to examine variations in the treatment of a nondisplaced, extraarticular distal radius fracture. The survey comprised 9 demographic questions and a clinical casevignette in which the patient had a nondisplaced extra-articular distal radius fracture (Appendix). Participants were asked to choose a treatment option for this patient, as well as the duration of immobilization if surgery was not recommended. Options for appropriate treatment included short arm casting, short arm/thumb spica casting, long arm casting, long arm/thumb spica casting, surgery, or other, with the option to specify details of an alternative treatment. The physicians were also asked to identify the number of times they personally treated this type of injury in the past year.
Surveys were distributed and collected over 8 months, from November 2012 through June 2013.

\section{Participants}

Inclusion criteria required that participants be graduates of an accredited medical school and practicing orthopedists at the time of survey distribution. Surveys were distributed in Article and electronic format. Physicians were recruited via E-mail, in-person visits to hospitals and orthopedic clinics primarily in the mid-Atlantic region of the United States, and distribution at the AAOS Annual Meeting held in Chicago in March 2013.

\section{Main Outcome Measures}

During analysis, treatment selections were categorized as surgical or nonsurgical interventions. In the event that "other" was selected as the appropriate treatment option, the comment field was used to categorize the intervention as surgical or nonsurgical. If the comment field specified any type of closed reduction or casting, the response was categorized as nonsurgical. For comments that gave a nonspecific or ambiguous alternative, such as referral to a specialist or determination based on patient preference or patient occupation, the response could not be deemed surgical or nonsurgical and was categorized as "other."

\section{Sample Size}

The appropriate sample size was calculated using a $95 \%$ confidence interval (CI) and a 5\% margin of error. An estimated prevalence of surgical interventions chosen as the appropriate treatment option of $50 \%$ was used to yield the most conservative estimate of sample size. The standard equation for determining sample size for a simple, random sample was then used to calculate a sample size of 380 respondents.

\section{Statistical Methods}

We used $\chi^{2}$ tests of independence to examine the association between physician demographic characteristics and surgery selection. A $4 \times 2 \chi^{2}$ test was used to compare physician age to surgical selection, US region of practice to surgical selection, and 4 isolated subspecialties to surgical selection. When comparing practice setting to surgical selection, a $3 \times 2 \chi^{2}$ test was used, followed by pairwise comparisons. CIs were calculated for the observed proportions of casting type and duration. 


\section{Cost Analysis}

Direct medical costs were estimated using published analysis of Medicare payments for beneficiaries aged $\geq 65$ years in $2007 .{ }^{10}$ Medicare payments should reflect relative cost differences between different procedures, and should, on average, exceed costs. ${ }^{11,12}$ Shauver et $\mathrm{a}^{10}$ obtained inpatient and outpatient Medicare claims files for fractures of the radius and/or ulna (International Classification of Diseases, 9th Revision, Clinical Modification [ICD-9-CM] codes 813.00 to 813.03) for 2007 from the Centers for Medicare and Medicaid Services. They then used the ICD-9-CM and Current Procedural Terminology codes to identify distal radius fractures as well as the specific treatment used. Linking the claims over an episode of care, they were able to allocate the payments by the source of service. Shauver et al estimated that Medicare paid, on average, $\$ 1,458.74$ for closed treatment and $\$ 3,832.17$ for internal fixation. However, the scenario used in the survey focused on a patient unlikely to be insured by Medicare. To the extent that private insurers pay more than Medicare, and assuming that Medicare payments can be used as a proxy for real resource costs, it is likely that health care spending will significantly exceed estimates of the direct costs.

Indirect medical costs reflect missed opportunities that may arise from medical visits, additional time needed to complete basic tasks, time of family and friends called on to provide assistance, and other foregone events, including time off from school or work because of injury and treatment. We assumed the following indirect costs: $80 \mathrm{~min}$ utes/physician visit, and, based on the duration of immobilization or casting, 15 minutes/day in activities of daily living, 60 minutes/week for missed opportunities and assistance from others, and 600 minutes/week in foregone productivity. The value of time was based on the average annual salary estimated by Social Security Administration actuaries, which for 2014 was $\$ 49,372.25 .^{13}$

\section{Ethical Review}

The Georgetown University Institutional Review Board, Washington, DC, reviewed and deemed this study exempt on August 31, 2012.

\section{Results}

Of the 494 survey responses received, 5 respondents did not meet the inclusion criteria as retired
Table 1. Characteristics of the Study Sample $(n=489)$

\begin{tabular}{|c|c|c|}
\hline \multirow[b]{2}{*}{ Characteristics } & \multicolumn{2}{|c|}{ Study Sample } \\
\hline & No. & $\%$ \\
\hline \multicolumn{3}{|l|}{ Age (years) } \\
\hline$<36$ & 66 & 13.6 \\
\hline $36-40$ & 82 & 16.8 \\
\hline $41-45$ & 79 & 16.2 \\
\hline $46-50$ & 55 & 11.3 \\
\hline $51-55$ & 61 & 12.5 \\
\hline $56-60$ & 66 & 13.6 \\
\hline$>60$ & 78 & 16.0 \\
\hline \multicolumn{3}{|l|}{ Sex } \\
\hline Male & 459 & 94.1 \\
\hline Female & 29 & 5.9 \\
\hline \multicolumn{3}{|l|}{ Setting } \\
\hline Private practice & 323 & 66.2 \\
\hline MedSpec clinic & 33 & 6.8 \\
\hline University & 113 & 23.2 \\
\hline Other & 79 & 16.2 \\
\hline \multicolumn{3}{|l|}{ Classification } \\
\hline $\begin{array}{l}\text { General orthopedic } \\
\text { surgeon }\end{array}$ & 168 & 34.4 \\
\hline Subspecialty orthopedist & 309 & 63.3 \\
\hline Other & 22 & 4.5 \\
\hline \multicolumn{3}{|l|}{ Subspecialty $(\mathrm{n}=309)$} \\
\hline Adult reconstruction & 16 & 5.2 \\
\hline Arthroscopy & 12 & 3.9 \\
\hline Foot/ankle & 62 & 20.1 \\
\hline Hand & 40 & 12.9 \\
\hline Elbow/upper extremity & 32 & 10.4 \\
\hline Hip/knee/lower extremity & 34 & 11.0 \\
\hline Joints & 20 & 6.5 \\
\hline Trauma & 31 & 10.0 \\
\hline Pediatrics & 24 & 7.8 \\
\hline Spine & 17 & 5.5 \\
\hline Sports medicine & 60 & 19.4 \\
\hline Other & 3 & 1.0 \\
\hline \multicolumn{3}{|l|}{ Country } \\
\hline United States & 328 & 69.5 \\
\hline Other & 144 & 30.5 \\
\hline \multicolumn{3}{|l|}{ Time spent with patients (\%) } \\
\hline$<50$ & 30 & 6.2 \\
\hline $50-75$ & 128 & 26.3 \\
\hline$>75$ & 321 & 65.9 \\
\hline
\end{tabular}

individuals and were eliminated from the analysis. Table 1 shows the demographic distribution of respondents. Respondents were 94\% male, which aligns with the current gender distribution in the orthopedic field. ${ }^{14,15}$ The majority of respondents identify themselves as working in private practice $(66 \%)$, as subspecialists (63\%), and as orthopedists 
Figure 1. Distribution of treatment selection. Long-arm casting includes long-arm spica casting, and short-arm casting includes short-arm spica casting. The "other" category in this case refers to all responses classified as nonsurgical interventions that did not specify long-arm or short-arm casting.

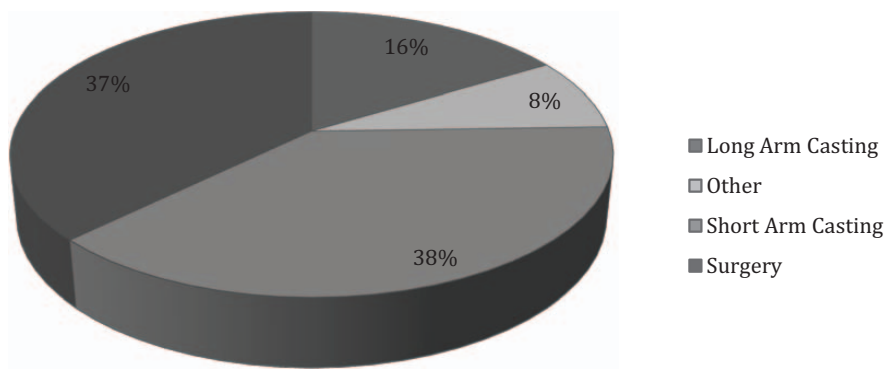

who spend $>75 \%$ of their professional time seeing patients (66\%). Approximately $70 \%$ of respondents currently practice medicine in the United States.

Treatment selection was not uniform. Figure 1 shows the distribution of surgical and nonsurgical interventions. Of the responses, $37 \%$ indicated a surgical intervention as the appropriate treatment (95\% CI, 32.7-41.3\%), whereas the remainder of the responses indicated a nonsurgical intervention or were not specific as to the nature of their treatment recommendation. The majority of nonsurgical selections specified short arm casting as the appropriate intervention (38\% of overall responses; $95 \%$ CI, $33.7-42.3 \%$ ), whereas $16 \%$ of respondents selected a long arm casting option (95\% CI, $12.8-19.3 \%)$.

Among respondents who identified themselves as subspecialty orthopedists, $41 \%$ selected a surgical intervention, whereas $36 \%$ of general orthopedists selected a surgical intervention $(P=.33$; Figure 2). The rate of surgical intervention varied significantly by age group $(P<.01$; Figure 2$)$. Respondents aged 40 to 49 years selected surgical intervention with a higher frequency than the other age groups $(49 \% ; P<.01)$, whereas respondents younger than age 40 selected surgical intervention with a lower frequency than other age groups $(29.5 \% ; P=.02)$. The frequency of surgical selection was similar between physicians practicing in the United States (38.0\%) and their non-US counterparts $(38.4 \%)$. Within the United States there was no significant regional difference in surgery selection rate $(P=.57$; regions were defined by US Census Bureau guidelines). The biggest regional difference was between the West (34\%) and South (39\%), though it was not statistically significant $(P=.24)$.

An association between practice setting and surgical selection was also found $(P=.04)$. Of respondents who indicated that they see patients primarily in multispecialty clinics, $52 \%$ selected surgery. The frequency of surgical selection was $40 \%$ among

Figure 2. Frequency of surgical treatment selection by age of physician and classification of physician. Average frequency was $37 \%$.

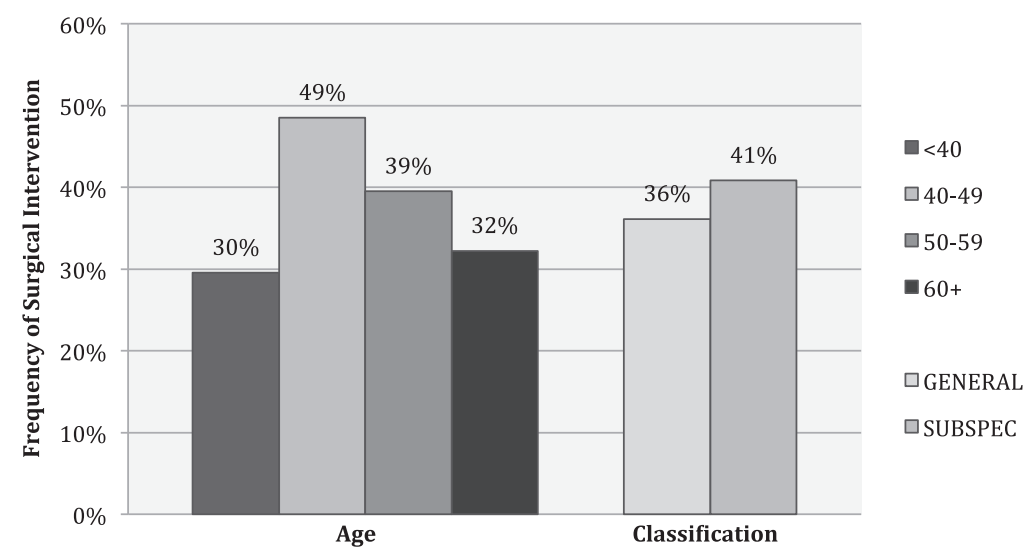


Figure 3. Frequency of surgical treatment selection by physician subspecialty. Average frequency was $37 \%$.

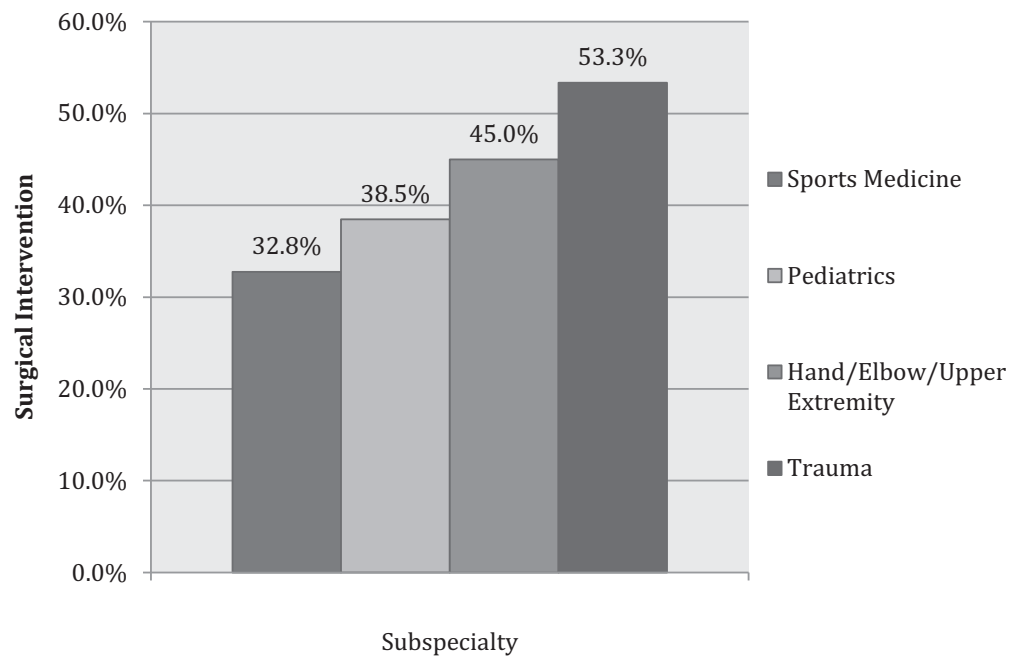

those who see patients primarily in private practice and $30 \%$ for those who primarily see patients in a university setting. Specifically, surgical selection in the university setting was significantly lower than in multispecialty clinics $(P=.02$ in a pairwise comparison) and significantly lower than in private practice $(P=.05)$.

Figure 3 shows the frequency of surgical treatment selections among 4 subspecialties: sports medicine (33\%), pediatrics (39\%), upper extremity (45\%), and trauma (53\%). These subspecialties were individually analyzed because of their clinical relevance regarding this treatment. No association was found between the rate of surgical selection and these subspecialties $(P=.27)$.

Duration of immobilization selections ranged from 2 to 12 weeks. The majority of respondents $(69 \%)$ indicated 6 weeks as the appropriate casting duration (95\% CI, 64.9-73.1\%), whereas $15 \%$ selected an immobilization period of $<6$ weeks and $9 \%$ selected $>6$ weeks. The remainder either wrote in a range or a combination of immobilization periods.

\section{Differences in Total Costs}

Surgery requires about $76 \%$ more resources (\$5228) than nonsurgical treatments $(\$ 2979)$, assuming a casting period of 5 weeks (Table 2). However, depending on how long the patient is in a cast, the indirect costs can vary considerably. Indirect costs for 8 weeks in a cast may be $57 \%$ greater than those for 5 weeks in a cast.

\section{Discussion}

Our findings suggest a lack of consistency in the treatment of extra-articular distal radius fractures as reported by orthopedic surgeons. Treatment selections ranged from conservative, nonoperative management to surgical treatment for an identical injury, suggesting the absence of a consensus among orthopedic surgeons with respect to the ideal management protocol for this injury at the broadest level.

Within the category of nonsurgical intervention, the decision between short and long arm casting also lacked consistency. Though the ma-

Table 2. Comparison of Average Resource Costs*

\begin{tabular}{lccr}
\hline & Casting & Surgery & Difference \\
\hline Direct medical costs & $\$ 1525$ & $\$ 4004$ & $163 \%$ \\
Visits/duration & 4 Visits/5 weeks & 4 Visits/4 weeks & \\
Indirect costs & $\$ 1454$ & $\$ 1223$ & $(16 \%)$ \\
Total costs & $\$ 2979$ & $\$ 5228$ & $76 \%$ \\
\hline
\end{tabular}

*Reimbursement from private insurance is likely to be considerably higher then these estimates of resource costs. 
jority called for short arm casting, the proportion selecting long arm casting (16\%) still contributed to the overall response distribution (95\% CI, $12.8-19.3 \%)$. The appropriate duration of immobilization ranged from 2 to 12 weeks, suggesting an arbitrary element in this facet of the treatment decision as well. Though a casting duration of 6 weeks was selected with the greatest frequency, other time frames comprised $>25 \%$ of the nonsurgical immobilization responses, and this choice can have a substantial impact on quality of life and cost of care.

Treatment selection was associated with physician age and practice setting. Specifically, associations were found between physicians $<40$ years old and those 40 to 49 years old and surgical intervention. Though we originally speculated that the younger generation of orthopedists would tend to treat surgically more often, the group $<40$ years of age was associated with a lower likelihood of surgical intervention, whereas the 40- to 49-year-old group was associated with a higher frequency of surgical intervention. We speculate that physicians older than 50 years may be more accustomed to casting and may stick to that form of treatment. Though we predicted that subspecialists would treat surgically more often, there was no association between identification as a general orthopedist or subspecialist and surgery selection, but our subgroup numbers were small. The differences in treatment were not associated with geographic variations as hypothesized, and were found to be uniform between US and non-US-based participants, and were not significantly different between regions of the United States. Practice setting, however, was associated with surgical intervention. Physicians who practice in a university setting were less likely to treat surgically than physicians who see patients in multispecialty clinics and their counterparts in private practice.

Assuming 640,000 distal radius fractures in a year, with the difference in direct cost between casting and surgical treatment being \$2249, a cost savings of $\$ 1.4$ billion would be appreciated if all patients were casted. ${ }^{1}$ This is clearly an overestimation, because casting is not indicated for all patients, but it highlights the magnitude of the difference in cost. All estimates exclude the cost of prescription drugs and costs associated with complications, which might increase direct medical costs another $2 \% .{ }^{10}$ Moreover, this is a conservative estimate of resource costs and not reimbursement, which likely would be considerably greater.

Limitations of this study include that we did not actually observe orthopedists in practice but queried their practice preferences outside of the clinical setting. We did this to standardize patient variables; we believed chart or database reviews would have too many variables that were not measureable. Anyone who practices medicine realizes there is an art to taking care of patients, and no 2 are identical, resulting in a variety of appropriate approaches. However, by providing physicians with the same vignette, we created an ideal situation in which, if a standard existed, it would be observed in our analysis. Surveys have limitations and inherent bias, but because of the sample size and the commonplace injury, we assumed generalizability. In addition, because of the nature of survey distribution, we were not able to obtain a response rate. We also relied on Medicare administrative data and the use of payments as a proxy for resource costs. However, to the extent that Medicare reimbursements are substantially lower than private insurers, payments for direct medical costs are likely to be considerably greater than assumed in this analysis.

Our goal was to examine clinical treatment practices of extra-articular, nondisplaced distal radius fracture treatment, with the presumption that orthopedic physicians have informally established a standard of care. Unfortunately, that is not the case, and it seems that largely diverse care is practiced, ranging from 2 weeks of casting to surgery. The lack of standardization greatly affects patients individually and society as whole because of the potential increased cost of surgery if it is not indicated and the impact of different casting durations on quality of life. We purposely chose a very common condition because we realize that there is much practice variation and a lack of high-level evidence for many procedures in medicine. One hopes, however, that there would be practice conformity for such a common procedure. It is our hope that funding agencies, stakeholders, and organizations can work toward improving this practice and bringing fracture 
care to the 21 st century, where evidence is easily obtainable with proper trials.

\section{References}

1. Chung KC, Spilson SV. The frequency and epidemiology of hand and forearm fractures in the United States. J Hand Surg Am 2001;26:908-15.

2. Bucholz RW, Heckman JD, Court-Brown CM. Rockwood and Green's fractures in adults. Philadelphia: Lippincott Williams \& Wilkins; 2006.

3. Lichtman DM, Bindra RR, Boyer MI, et al. Treatment of distal radius fractures. J Am Acad Orthop Surg 2010;18:180-9.

4. Handoll HH, Madhok R. Conservative interventions for treating distal radial fractures in adults. Cochrane Database Syst Rev 2003;(2):CD000314.

5. McAuliffe TB, Hilliar KM, Coates CJ, Grange WJ. Early mobilisation of Colles' fractures. A prospective trial. J Bone Joint Surg Br 1987;69:727-9.

6. Vang Hansen F, Staunstrup H, Mikkelsen S. A comparison of 3 and 5 weeks immobilization for older type 1 and 2 Colles' fractures. J Hand Surg 1998;23: $400-1$.

7. Moseley JB, O'Malley K, Petersen NJ, et al. A controlled trial of arthroscopic surgery for osteoarthritis of the knee. N Engl J Med 2002;347:81-8.

8. Smith R. Where is the wisdom. . .? BMJ 1991;303: 798-9.

9. Kumar S, Nash D. Health care myth busters: is there a high degree of scientific certainty in modern medicine? Scientific American, March 25, 2011. Available from: http://www.scientificamerican.com/article/ demand-better-health-care-book/. Accessed January 26, 2016.

10. Shauver MJ, Yin H, Banerjee M, Chung KC. Current and future national costs to medicare for the treatment of distal radius fracture in the elderly. J Hand Surg 2011;36:1282-7.

11. Hayes KJ, Pettengill J, Stensland J. Getting the price right: Medicare payment rates for cardiovascular services. Health Aff (Millwood) 2007;26:124-36.

12. Newhouse JP. Pricing the priceless: a health care conundrum. Cambridge, MA: MIT Press; 2004.

13. Office of the Chief Actuary, Social Security Administration. The long-range demographic assumptions for the 2013 Trustees Report. May 2013. Available from: https://www.ssa.gov/oact/tr/2013/2013_LongRange_Demographic_Assumptions.pdf. Accessed January 26, 2016.

14. O'Connor MI. Orthopedic surgery: women on the rise in a male-dominated field. Huffington Post, August 2, 2012. Available from: http://www.huffingtonpost.com/dr-mary-i-oconnor/orthopedic-surgerywomen_b_1726145.html. Accessed January 26, 2016.
15. Porucznik M. Where are the women orthopaedists? AAOS Now, February 2008. Available from: http:// www.aaos.org/AAOSNow/2008/Feb/cover/cover2/ ?ssopc $=1$. Accessed January 26, 2016.

\section{Appendix}

Survey of Appropriate Fracture Treatment

1. What is your age? years

2. Sex: $\square$ male $\square$ female

3. When did you graduate from medical school? Year:

4. In which country did you attend medical school?

5. What percentage of your professional time is spent seeing patients?

6. Where do you currently practice medicine?

City: State:

Country:

7. In what setting do you see patients?

$\square$ Private practice $\square$ MedSpec clinic $\square$ University Other

8. Which of the following do you consider yourself?

$\square$ General orthopedic surgeon $\square$ Subspecialty orthopedist (Specialty: ) $\square$ Other

9. How many fractures do you treat annually? fractures

\section{Vignette}

10. MC is a 42-year-old woman with no significant medical history who presents to the emergency department with right wrist pain. She tripped over a curb while walking her dog, falling on an outstretched hand. Immediate swelling and deformity developed, and pain was noted to be 9 of 10, sharp, and throbbing.

Physical examination is significant for gross deformity, swelling, and ecchymosis of the right wrist. She has significant tenderness at the site of deformity. Patient is neurovascularly intact. Radiographs demonstrate a displaced, extra-articular distal radius fracture. There is no dorsal comminution. An orthopedic consult is requested and closed reduction with splinting is performed. Repeat radiographs show near-normal alignment of the distal radius.

One week later, MC presents to the orthopedic clinic for follow-up. Her pain is well controlled and she has been compliant with not bearing weight while in the splint. 
Radiographs at this time show no further displacement of the extra-articular distal radius fracture.

Appropriate treatment at this time would be:

$\square$ Short arm/thumb spica casting $\square$ Long arm/ thumb spica casting

$\square$ Short arm casting $\square$ Long arm casting
Surgery $\square$ Other:

Appropriate duration of casting (if selected):

$\square 4$ weeks $\square 6$ weeks

$\square 8$ weeks $\square 12$ weeks

$\square$ Other:

How often in the past year did you treat a fracture like this? times 